INPLASY

PROTOCOL

To cite: Li et al. Percutaneous endoscopic versus minimally invasive transforaminal lumbar interbody fusion for lumbar degenerative diseases: a meta-analysis. Inplasy protocol 202220090. doi:

10.37766/inplasy2022.2.0090

Received: 21 February 2022

Published: 21 February 2022

Corresponding author:

Yu-Fei Fu

fuyufei1985@163.com

Author Affiliation:

Xuzhou Central Hospital

Support: None.

Review Stage at time of this submission: Preliminary searches.

Conflicts of interest:

None declared.

\section{Percutaneous endoscopic versus} minimally invasive transforaminal lumbar interbody fusion for lumbar degenerative diseases: a meta-analysis

Review question / Objective: Transforaminal lumbar interbody fusion (TLIF) has been widely used for patients with lumbar degenerative disease (LDD). The most common used techniques include minimally invasive TILF (MIS-TILF) and percutaneous endoscopic TLIF (PE-TLIF).

Eligibility criteria: The inclusion criteria were as follows: Studies: comparative studies of PE-TLIF and MIS-TLIF; Diseases: LDD;Languages: Not limited.The exclusion criteria were as follows:(a) single-arm studies; (b) non-human studies; and (c) studies without English title and/or abstract.

INPLASY registration number: This protocol was registered with the International Platform of Registered Systematic Review and Meta-Analysis Protocols (INPLASY) on 21 February 2022 and was last updated on 21 February 2022 (registration number INPLASY202220090).

\section{INTRODUCTION}

Review question / Objective: Transforaminal lumbar interbody fusion (TLIF) has been widely used for patients with lumbar degenerative disease (LDD). The most common used techniques include minimally invasive TILF (MIS-TILF) and percutaneous endoscopic TLIF (PETLIF).
Condition being studied: Lumbar degenerative disease (LDD), which include lumbar spinal stenosis, lumbar disc herniation, spondylolisthesis, and lumbar instability, are common causes of back and leg pain. Transforaminal lumbar interbody fusion (TLIF) has been widely used for patients with LDD and the purposes of TLIF are relieving the symptoms and improving the quality of life. However, traditional open TLIF is associated with iatrogenic injury of 
paraspinal muscle, which could cause postoperative intractable low back pain. At present, minimally invasive TILF (MIS-TILF) has been widely used to reduce the soft tissue injury and intraoperative blood loss. The MIS-TLIF has showed significantly less blood loss compared with open TLIF. However, MIS-TLIF is usually limited by a narrow operating space and it may be difficult to view the deeper surgical field through the tubular retractor. To overcome the shortcomings of MIS-TLIF, percutaneous endoscopic TLIF (PE-TLIF) has been commonly used for patients with LDD. PE-TLIF can achieve fully endoscopic discectomy, decompression of the spinal canal and foramina, and interbody fusion through the endoscopic and working portal. While there have been multiple prior studies comparing the relative effectiveness of PE-TLIF and MIS-TLIF as treatments for LDD, most of these analyses are retrospective in design. Given that many factors can influence the results of a given retrospective study, potentially resulting in unintentional result bias, a meta-analysis is warranted to systematically survey these studies and to lower the risk of such bias while increasing the overall statistical power beyond that which can be achieved through smaller studies.

\section{METHODS}

Search strategy: ((Endoscopic Lumbar Interbody Fusion) OR (Endo-LIF)) AND ((Minimally Invasive Transforaminal Lumbar Interbody Fusion) OR (MIS-TLIF)).

Participant or population: LDD patients.

Intervention: PE-TLIF.

\section{Comparator: MIS-TLIF.}

Study designs to be included: The inclusion criteria were as follows: Studies: comparative studies of PE-TLIF and MISTLIF; Diseases: LDD; Languages: Not limited.The exclusion criteria were as follows:(a) single-arm studies; (b) nonhuman studies; and (c) studies without English title and/or abstract.
Eligibility criteria: The inclusion criteria were as follows: Studies: comparative studies of PE-TLIF and MIS-TLIF; Diseases: LDD;Languages: Not limited.The exclusion criteria were as follows:(a) single-arm studies; (b) non-human studies; and (c) studies without English title and/or abstract.

Information sources: Relevant articles were searched in PubMed, Embase, Cochrane Library, Wanfang, VIP, and CINK.

Main outcome(s): The endpoints of this meta-analysis included visual analog scale (VAS)-leg, VAS-back, Oswestry Disability Index (ODI), fusion rate, operation time, blood loss, complication rates, and postoperative hospital stay.

Data management: RevMan v5.3 and Stata 12.0 were used in this study. Dichotomous variables are pooled depending upon odds ratios (ORs) with $95 \%$ confidence intervals (Cls), while continuous variables are pooled depending upon mean difference (MD) with $95 \%$ Cls. Heterogeneity is assessed by 12 tests, with $\mathrm{I} 2>50 \%$ suggesting significant heterogeneity. Random-effects models are employed for significant heterogeneity, while fixed-effects models are employed for significant homogeneity. Heterogeneity sources are analyzed through sensitivity assessment. Funnel plot and Egger test are employed for evaluating publication bias risks.

Quality assessment / Risk of bias analysis: Randomized controlled trials (RCTs) were assessed by the Cochrane risk of bias tool for the potential bias in performance, attrition, detection, selection, reporting, and others. Non-RCTs were assessed by the 9-point Newcastle-Ottawa scale (NOS) for potential bias in selection (4 points), comparability ( 2 points), and exposure ( 3 points), with assessments of high $(\geq 7)$, moderate (4-6), and low (<4) risk of bias.

Strategy of data synthesis: RevMan v5.3 and Stata 12.0 were used in this study. Dichotomous variables are pooled depending upon odds ratios (ORs) with $95 \%$ confidence intervals (Cls), while 
continuous variables are pooled depending upon mean difference (MD) with $95 \%$ Cls. Heterogeneity is assessed by 12 tests, with $12>50 \%$ suggesting significant heterogeneity. Random-effects models are employed for significant heterogeneity, while fixed-effects models are employed for significant homogeneity. Heterogeneity sources are analyzed through sensitivity assessment. Funnel plot and Egger test are employed for evaluating publication bias risks.

Subgroup analysis: None.

Sensitivity analysis: None.

Language: English.

Country(ies) involved: China.

Keywords: Transforaminal lumbar interbody fusion; Lumbar degenerative disease; Oswestry Disability Index; Metaanalysis.

Contributions of each author:

Author 1 - Jie Li.

Author 2 - Yu-Fei Fu. 